\title{
Evidence for a toroidal magnetic-field component in 5C 4.114 on kiloparsec scales (Research Note)
}

\author{
Denise C. Gabuzda ${ }^{1}$, Sebastian Knuettel ${ }^{1}$, and Annalisa Bonafede ${ }^{2}$ \\ 1 Department of Physics, University College Cork, Cork, Ireland \\ e-mail: d.gabuzda@ucc.ie, s.knuettel@mars.ucc.ie \\ 2 Hamburger Sternwarte, Gojenbergsweg 112, 21029 Hamburg, Germany \\ e-mail: annalisa. bonafede@hs .uni-hamburg.de
}

Received 13 August 2015 / Accepted 23 September 2015

\begin{abstract}
Context. A monotonic, statistically significant gradient in the observed Faraday rotation measure (RM) across the jet of an active galactic nucleus (AGN) reflects a corresponding gradient in the electron density and/or line-of-sight magnetic (B) field in the region of Faraday rotation. For this reason, such gradients may indicate the presence of a toroidal B field component, possibly associated with a helical jet B field. Although transverse RM gradients have been reported across a number of parsec-scale AGN jets, the same is not true on kiloparsec scales, suggesting that other (e.g. random) magnetic-field components usually dominate on these larger scales. Aims. We wished to identify clear candidates for monotonic, transverse RM gradients across AGN jet and lobe structures on scales larger than those probed thus far, and estimate their statistical significances.

Methods. We identified an extended, monotonic transverse Faraday-rotation gradient across the northern lobe of a previously published Very Large Array (kiloparsec-scale) RM image of 5C 4.114. We reanalyzed these VLA data in order to determine the significance of this RM gradient.

Results. The RM gradient across the northern kiloparsec-scale lobe structure of 5C 4.114 has a statistical significance of about $4 \sigma$. There is also a somewhat less prominent monotonic transverse Faraday-rotation gradient across the southern jet/lobe (narrower range of distances from the core, significance $\simeq 3 \sigma$ ). Other parts of the Faraday-rotation distribution observed across the source are patchy and show no obvious order.

Conclusions. This suggests that we are observing a random RM component associated with the foreground material in the cluster in which the radio source is located and through which it is viewed, superposed on a more ordered RM component that arises in the immediate vicinity of the AGN jets. We interpret the transverse RM gradient as reflecting the systematic variations of the line-of-sight component of a helical or toroidal B field associated with the jets of 5C 4.114. These results suggest that the helical field that arises due to the joint action of the rotation of the central black hole and its accretion disc and the jet outflow can survive to distances of thousands of parsec from the central engine.
\end{abstract}

Key words. magnetic fields - polarization - galaxies: jets

\section{Introduction}

The radio emission of radio galaxies and active galactic nuclei (AGNs) is synchrotron emission, which can be linearly polarized up to about $75 \%$ in optically thin regions, where the polarization angle $\chi$ is orthogonal to the projection of the magnetic field $\boldsymbol{B}$ onto the plane of the sky (Pacholczyk 1970). Linear polarization measurements thus provide direct information about both the degree of order and the direction of the $\boldsymbol{B}$ field giving rise to the observed synchrotron radiation.

Multi-frequency interferometric polarization observations also provide high-resolution information about the distribution of the Faraday rotation of the observed polarization angles arising between the source and observer. When the Faraday rotation occurs outside the emitting region in regions of non-relativistic plasma, the amount of rotation is given by

$\chi_{\mathrm{obs}}-\chi_{\mathrm{o}}=\frac{e^{3} \lambda^{2}}{8 \pi^{2} \epsilon_{\mathrm{o}} m^{2} c^{3}} \int n_{e} \boldsymbol{B} \cdot \mathrm{d} \boldsymbol{l} \equiv R M \lambda^{2}$ where $\chi_{\mathrm{obs}}$ and $\chi_{\mathrm{o}}$ are the observed and intrinsic polarization angles, respectively, $-e$ and $m$ are the charge and mass of the particles giving rise to the Faraday rotation, usually taken to be electrons, $c$ is the speed of light, $n_{\mathrm{e}}$ is the density of the Faraday-rotating electrons, $\boldsymbol{B} \cdot \mathrm{dl}$ is an element of the line-ofsight magnetic field, $\lambda$ is the observing wavelength, and RM (the coefficient of $\lambda^{2}$ ) is the rotation measure (RM; e.g., Burn 1966). Simultaneous multifrequency observations thus allow the determination of both the RM, which carries information about the electron density and $\boldsymbol{B}$ field in the region of Faraday rotation, and $\chi_{0}$, which carries information about the intrinsic $\boldsymbol{B}$-field geometry associated with the synchrotron source.

Systematic gradients in the Faraday rotation have been reported across the parsec-scale jets of a number of AGN, interpreted as reflecting the systematic change in the line-of-sight component of a toroidal or helical jet $\boldsymbol{B}$ field across the jet (e.g., Hovatta et al. 2012; Mahmud et al. 2013; and Gabuzda et al. 2014b, 2015). Such fields would come about in a natural way as a result of the "winding up" of an initial "seed" field 
by the differential rotation of the central accreting objects (e.g. Nakamura et al. 2001; Lovelace et al. 2002).

It is an interesting question whether these ordered fields can survive to larger scales as the jets propagate outward. The presence of a clear transverse RM gradient on scales of more than a hundred parsec from the jet base was reported for 3C 380 (Gabuzda et al. 2014a). However, attempts to search for possible kiloparsec-scale transverse Faraday-rotation gradients across the jet structures of extragalactic radio sources by eye have yielded only a small number of candidates (Gabuzda et al. 2012). This is consistent with a picture in which random distributions of the $\boldsymbol{B}$ field and electron density in the general vicinity of the radio source (e.g., in the cluster or inter-cluster medium in which the source is located) generally dominate on these large scales. Only one claim of a transverse Faraday-rotation gradient that could be associated with a helical $\boldsymbol{B}$ field present on kiloparsec scales has been made (Kronberg et al. 2011).

We present here a new analysis of observations of the radio galaxy $5 \mathrm{C} 4.114$, whose position is approximately coincident with the location of the Coma cluster of galaxies, originally obtained and analyzed by Bonafede et al. (2010), which demonstrates the presence of monotonic, statistically significant Faraday-rotation gradients across both the northern lobe and southern jet/lobe. The northern gradient is especially striking. If associated with the azimuthal components of helical $\boldsymbol{B}$ fields, the orientation of the gradients is consistent with the initial $\boldsymbol{B}$ field that is wound up having a dipolar-like configuration.

\section{Observations and reduction}

The data used for our analysis are precisely the $1.365,1.516$, 4.535 and $4.935 \mathrm{GHz}$ Very Large Array data considered by Bonafede et al. (2010), and the observations and the data calibration and reduction methods used are described in that paper.

We could not use the RM map published by Bonafede et al. (2010) directly, because the associated error map did not take into account the finding of Hovatta et al. (2012) that the uncertainties in the Stokes $Q$ and $U$ fluxes in individual pixels onsource are somewhat higher than the off-source rms fluctuations, potentially increasing the resulting RM uncertainties.

To address this, we imported the final, fully self-calibrated visibility data of Bonafede et al. (2010) into the AIPS package, then used these data to make naturally weighted $I, Q$ and $U$ maps at all four frequencies, with matching image sizes, cell sizes and beam parameters specified by hand in the AIPS task IMAGR. These images were all convolved with a circular Gaussian beam having a full-width at half-maximum of $1.3^{\prime \prime}$. We then obtained maps of the polarization angle, $\chi=\frac{1}{2} \arctan (U / Q)$, and used these to construct corresponding maps of the Faraday RM using the AIPS task RM. The uncertainties in the polarization angles used to obtain the RM fits were calculated from the uncertainties in $Q$ and $U$, which were estimated using the approach of Hovatta et al. (2012). The output pixels in the RM maps were blanked when the RM uncertainty resulting from the $\chi$ vs. $\lambda^{2}$ fits exceeded $8 \mathrm{rad} / \mathrm{m}^{2}$.

\section{Results}

Figure 1 presents our 4.535-GHz intensity image of 5C 4.114, with the RM image superposed in colour; these essentially reproduce the images in Fig. 12 of Bonafede et al. (2010).

A monotonic gradient in the RM across the northern radio lobe is clearly visible by eye, highlighted by the upper black arrow in Fig. 1. A less prominent, oppositely directed RM gradient is visible across the southern jet/lobe, highlighted by the lower black arrow in Fig. 1. Both regions are fairly well resolved, and span two to three beamwidths in the transverse direction. The ordered RM gradient crossing the northern lobe is quite unusual for the kiloparsec-scale Faraday rotation distributions of radio galaxies and quasars, which tend to be more patchy.

The redshift of 5C 4.114 is not known, but no optical identification with either a Coma cluster galaxy nor with a background galaxy has been found, indicating that the source's redshift is greater than 0.023 (Bonafede et al. 2010). This indicates that the projected distance from the AGN core to the location of the northern transverse RM gradient is at least $2 \mathrm{kpc}$ (assuming a Hubble constant $H_{0}=71 \mathrm{~km} \mathrm{~s}^{-1} \mathrm{Mpc}^{-1}, \Omega_{\Lambda}=0.73$, and $\Omega_{\mathrm{m}}=0.27$ ), with the projected distance to the southern gradient being somewhat smaller.

\subsection{Significance of the transverse RM gradients}

Monotonic transverse RM gradients are observed throughout the regions enclosed by the grey boxes in Fig. 1; each of the points in the right-hand panels of Fig. 1 corresponds to a monotonic transverse RM gradient at some distance from the core. The uncertainties of the RM values were determined using $\chi$ uncertainties estimated in individual pixels using the approach of Hovatta et al. (2012), without including the effect of uncertainty in the EVPA calibration, since this cannot introduce spurious RM gradients (Mahmud et al. 2009; Hovatta et al. 2012). The uncertainty of the difference between the RM values at the two ends of a slice was estimated by adding the uncertainties for the two RM values in quadrature. Comparisons of the RM values at the two ends of the RM slices considered in Fig. 1 indicate that the northern and southern transverse gradients have significances reaching $4.2 \sigma$ and $3.6 \sigma$, respectively.

The significance of the transverse RM gradients across the northern lobe (upper right plot in Fig. 1) is $\simeq 3-4.2 \sigma$ throughout nearly the entire region considered, and everywhere exceeds $2 \sigma$. This is thus a quite extended and coherent RM structure. The significance of the less prominent transverse RM gradients across the southern jet/lobe (lower right plot in Fig. 1) is greater than $2 \sigma$ throughout most of the region enclosed by the southern grey box in Fig. 1, and reaches $\simeq 3-3.6 \sigma$ in the vicinity of the transverse black arrow in Fig. 1 .

\section{Discussion}

\subsection{Ability to detect the transverse RM gradients}

It is important to note here that we are interested in establishing whether the transverse RM gradients visible by eye represent a statistically significant, systematic, monotonic change in the observed Faraday RM across the lobe/jet structure. We are not interested in measuring the intrinsic RM values, only in investigating the reality of the observed gradients.

The question of the resolution necessary to reliably detect the presence of a transverse RM gradient has been discussed in the literature in the context of VLBI-scale RM measurements. Taylor \& Zavala (2010) had proposed that the reliable detection of a transverse RM gradient required that the observed RM gradient span at least three "resolution elements" (usually taken to mean three beamwidths) across the jet. This was tested by Mahmud et al. (2013) using Monte Carlo simulations based on model core-jet-like sources with transverse RM gradients present across their structures, which had intrinsic jet widths of $1 / 2,1 / 3,1 / 5,1 / 10$ and $1 / 20$ of the beam full-width 



Fig. 1. Left: 4.9-GHz intensity map of the 5C 4.114 with the RM distribution superposed. The intensity peak is $3.6 \mathrm{mJy} / \mathrm{beam}$, the lowest intensity contour is $5.0 \times 10^{-2} \mathrm{mJy} /$ beam, and the contours increase in increments of a factor of two. The two black arrows highlight transverse RM gradients visible across the northern lobe and the southern jet/lobe. The grey boxes show the regions for which the significances of series of parallel, monotonic transverse RM gradients are plotted in the right-hand panels; the grey arrows pointing outward along the jet show the direction of increasing pixel number in the right panels, and pixel 0 corresponds to the inner edge of each of the grey boxes. The pixel size is 0.1 arcsec. The $2 \sigma$ level is shown by the dashed blue horizontal lines, and the $3 \sigma$ level by the dashed red horizontal lines.

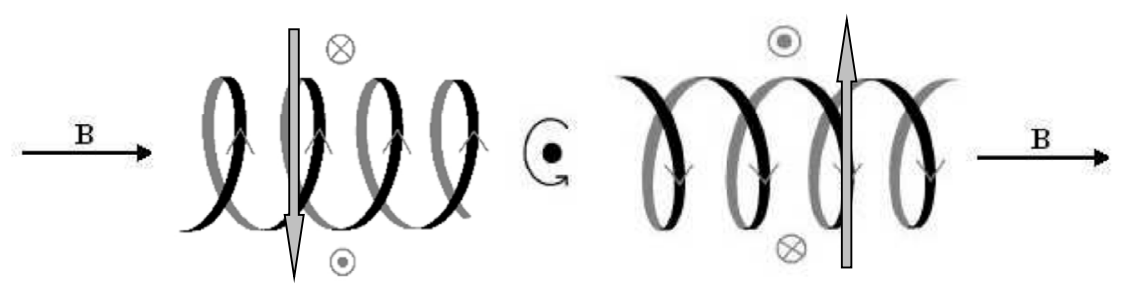

Fig. 2. Schematic of the effect of winding up a dipole-like poloidal $\boldsymbol{B}$ field (shown by the vertical arrows) by rotation of the central black hole and accretion disk. The circled dots and circled X's show the resulting azimuthal $\boldsymbol{B}$ field oriented towards and away from the observer on either side of the jets, and the filled grey arrows show the direction of the associated RM gradients.

at half-maximum (FWHM) in the direction across the jet. The resulting simulations show that the transverse RM gradients introduced into the model visibility data remained visible in RM maps constructed from realistic "noisy" data using standard techniques, even when the intrinsic width of the jet structure was much smaller than the beam width.

These Monte Carlo simulations (and also those of Hovatta et al. 2012) thus directly demonstrate that the three-beamwidth criterion of Taylor \& Zavala (2010) is overly restrictive, and that it is not necessary or meaningful to place a limit on the width spanned by an RM gradient in order for it to be reliable: the key criteria are that the gradient be monotonic and that the difference between the RM values at either end be at least $3 \sigma$. The counterintuitive result that a transverse RM gradient spanning only one beamwidth can potentially be significant essentially comes about because polarization is a vector quantity, while the intensity is a scalar. Thinking of the polarization as being composed of Stokes $Q$ and $U$, this enhanced sensitivity to closely spaced structures comes about because both $Q$ and $U$ can be positive or negative. Again, we are not speaking here of being able to accurately deconvolve the observed RM profiles to determine the intrinsic transverse RM structure - only of the ability to detect the presence of a systematic transverse RM gradient.

This means that we can consider the presence of the RM gradients detected across the northern lobe and southern jet/lobe of 5C 4.114, which are monotonic, encompass a range of RM values of at least $3 \sigma$, and span $\simeq 2-3$ beamwidths across the jet and $\simeq 0.5-1.5$ beamwidths along the jet, to be reliably detected, although it is not possible to derive the intrinsic (infinte-resolution) values of the gradients. The three-dimensional structures of the emission regions where the RM gradients are observed cannot be determined with certainty, although the general symmetry of 
the radio structure observed suggests that both the northern and southern jet/lobe structures are not very far from the plane of the sky.

\subsection{Random or ordered RM distributions on kpc scales?}

It is generally believed that the RM distributions across extragalactic radio sources on kiloparsec scales should be dominated by fairly random distributions of the electron density and $\boldsymbol{B}$ field in plasma that is not closely related to the radio source itself, for example, plasma that is associated with the cluster or intercluster medium. This view is supported by the fact that most of the observed RM distributions appear irregular and "patchy" (e.g., the RM maps presented by Bonafede et al. 2010 and Govoni et al. 2010). This was the framework in which the original study of Bonafede et al. (2010), aimed at constraining the Coma cluster magnetic-field strength, was carried out.

Based on their results for the seven FRI radio sources they considered, Bonafede et al. (2010) concluded that the observed RM distributions generally did not originate in the immediate vicinity of the sources, arising instead in intervening material of the Coma cluster. This conclusion was based on statistical analyses of the RMs and their uncertainties for different lines of sight through the cluster, and did not take into consideration possible patterns in the RM distributions for the individual objects studied.

However, the conclusions of Bonafede et al. (2010) for their sample as a whole and our own conclusions specifically for 5C 4.114 need not be in contradiction. We suggest that, indeed, the observed RM distributions of extragalactic radio sources on kiloparsec scales are usually (though not always) determined by intervening magnetised plasma that is not directly related to the jets; this plasma is not uniform and possibly turbulent, giving rise to an irregular, patchy RM distribution that bears no obvious relationship to the source structure. However, the resulting irregular RM distribution can be superposed on a more ordered RM distribution brought about by magnetised plasma in the immediate vicinity of the jet structure. Although it is usually the irregular RM component that is dominant, the ordered RM component may occasionally be visible in some individual objects. We suggest that this is the case for $5 \mathrm{C} 4.114$.

\subsection{Tentative evidence for a dipole-like initial field structure}

The transverse RM gradient detected across the southern jet/lobe structure of 5C 4.114 is somewhat less prominent than the northern RM gradient, which is very firmly detected. In this section, we explore the implications of the overall RM-gradient structure if both the (firm) northern and (somewhat tentative) southern RM gradients reflect the azimuthal (toroidal) component of a helical jet $\boldsymbol{B}$ field, brought about by the combination of the rotation of the central black hole and its accretion disk and the jet outflow. In this case, the directions of the transverse RM gradients on the sky imply particular directions for the associated azimuthal $\boldsymbol{B}$-field components; this, in turn, enables us to infer whether the poloidal components of the "seed fields" that were wound up by the rotation of the central black hole and accretion disk had the same or opposite senses in the two jets.

The direction of the azimuthal field component is determined by the direction of the central rotation and the direction of the poloidal component of the initial field that is "wound up". As is illustrated schematically in Fig. 3, the pattern shown by $5 \mathrm{C} 4.114$ is consistent with the poloidal $\boldsymbol{B}$-field component being directed outward in one jet and inward in the other, as would be expected for a dipolar-like initial $\boldsymbol{B}$-field configuration.

\section{Conclusion}

We have reconstructed the Faraday RM image of 5C 4.114 initial published by Bonafede et al. (2010) in order to quantitatively analyze various gradients visible in the RM image.

Our analysis has demonstrated that the differences in the RM values encompassed by the monotonic RM gradients visible across the entire northern lobe of the radio source and a more restricted region in the southern jet/lobe both exceed $3 \sigma$, making them statistically significant. The detection of the RM gradient across the northern lobe is very firm, while the RM gradient across the southern jet/lobe is slightly more tentative, due to the relatively narrow range of distances from the central AGN where the significance of the gradient exceeds $3 \sigma$.

This represents firm evidence that the northern, and possibly also the southern, kiloparsec-scale jet of 5C 4.114 carries a helical or toroidal $\boldsymbol{B}$-field component. Such a component would naturally arise due to the rotation of the central black hole and its accretion disc; apparently, this helical $\boldsymbol{B}$-field component can sometimes survive to distances of thousands of parsec from the central engine. Regardless of whether the regions where the RM gradients are observed represent outwardly propagating jets or lobes of material flowing backward toward the center of activity, they could contain the imprint of a helical $\boldsymbol{B}$-field component that was initially carried outward by the jet outflow. The relative orientations of the northern and southern gradients are consistent with the pattern expected if the initial poloidal jet $\boldsymbol{B}$ field had a dipole-like structure.

Our new analysis together with the original analysis of Bonafede et al. (2010) suggest a picture in which the observed RM distributions of extragalactic radio sources on kiloparsec scales are usually determined by intervening inhomogeneous, possibly turbulent magnetised plasma that is not directly related to the jets, with a more ordered RM distribution associated with magnetised plasma in the immediate vicinity of the jet structure occasionally becoming dominant in some regions of individual sources. This latter, ordered RM component can bear the imprint of a helical magnetic field associated with the jet structure.

\section{References}

Bonafede, A., Feretti, L., Murgia, M., et al. 2010, A\&A, 513, A30 Burn, B. J. 1966, MNRAS, 133, 67

Gabuzda, D. C., Christodoulou, D. M., Contopoulos, I. \& Kazanas, D. 2012, J. Phys.: Conf. Ser., 355, 012019

Gabuzda, D. C., Cantwell, T. M. \& Cawthorne, T. V. 2014a, MNRAS, 438, L1

Gabuzda D. C., Reichstein A. R. \& O'Neill E. L. 2014b, MNRAS, 444, 172

Gabuzda, D. C., Knuettel, S., \& Reardon, B. 2015, MNRAS, 450, 2441

Govoni, F., Dolag, K., Murgia, M., et al. 2010, A\&A, 522, A105

Hovatta, T., Lister, M. L., Aller, M. F., et al. 2012, AJ, 144, 105

Kronberg, P. P., Lovelace, R. V. E., Lapenta, G., \& Colgate, S. A. 2011, ApJ, 741, L15

Lovelace, R. V. E., Li, H., Koldoba, A. V., Ustyugova, G. V. \& Romanova, M. M. 2002, ApJ, 572, 445

Mahmud, M., Gabuzda, D. C., \& Bezrukovs, V. 2009, MNRAS, 400, 2

Mahmud, M., Coughlan, C. P., Murphy, E., Gabuzda, D. C., \& Hallahan, R. 2013, MNRAS, 431, 695

Nakamura, M., Uchida, Y., \& Hirose, S. 2001, New Astron., 6, 61

Pacholczyk, A. G., 1970, Radio Astrophysics (San Franciso: W. H. Freeeman)

Taylor, G. B., \& Zavala, R. 2010, ApJ, 722, L183 